\section{L.-S. Wang W.-T. Chou \\ Institute of Applied Mechanics, National Taiwan University, Taipei, Taiwan R.0.C.}

\title{
The Analysis of Constrained Impulsive Motion
}

Impulsive problems for mechanical systems subject to kinematic constraints are discussed in this paper. In addition to the applied impulses, there may exist suddenly changed constraints, or termed impulsive constraints. To describe the states of the system during the impulsive motion, three different phases, i.e., prior motion, virtual motion, and posterior motion, are defined which are subject to different sets of constraints, and thus have different degrees-of-freedom. A fundamental principle, i.e., the principle of velocity variation, for the constrained impulsive motion is enunciated as a foundation to derive the privileged impulse-momentum equations. It is shown that for a system with no applied impulse, a conservation law can be stated as the conservation of the virtual-privileged momenta. The proposed methodology provides a systematic scheme to deal with various types of impulsive constraints, which is illustrated in the paper by solving the constrained impulsive problems for the motion of a sleigh. [DOI: 10.1115/1.1577599]

\section{Introduction}

A mechanical system undergoes an impulsive motion if the motion, particularly the velocities, of the particles in the system are changed so rapidly that the duration of the process may be regarded to be instantaneous. Such phenomenon occurs when the system is subject to applied impulses or the constraints on the system are suddenly changed, and the associated problems are termed the constrained impulsive problems. In contrast to those continuously imposed constraints, termed the regular constraints, the suddenly varied constraints, called the impulsive constraints, may significantly affect the analysis of the impulsive motion, in which even the degree-of-freedom may be changed. How to systematically perform the analysis of the constrained impulsive motion subject to various types of impulsive constraints is the main theme of this paper.

Impulsive problems occur frequently in the motion of a mechanical system, and have been treated in many classical textbooks, such as [1-6], or in some recent literatures [7-12], among others. They also appear in the study of the motion subject to one-sided constraints, cf. [13-15] and the references therein, in which the continuous motion and jumps of kinematic variables exist hybridly. Analysis of such hybrid system, [16,17], requires an effective tool to deal with both the regular constraints and the impulsive constraints. According to their durations, the latter is further divided in this paper into four types, i.e. live, inert, elastic, and released. Similar classifications can be found in [1], but in which the live impulsive constraints are not mentioned and the released constraints have different characteristics. On the other hand, while the live constraints, as well as the inert ones, were discussed in $[3,8,18,19]$, the effects of released and elastic impulsive constraints have not been extensively analyzed. Although the mathematical formulation for impulsive constraints has been attempted in $[12,15,20,21]$, a synthesized approach to treat the above-mentioned four categories of impulsive constraints is still in demand. To attain this goal, a fundamental principle for impulsive motion needs to be invoked.

In 1903, Appell [1] enunciated a basic principle for percussion or impulsive motion analogous to D'Alembert's principle in dy-

Contributed by the Applied Mechanics Division of THE AMERICAN SOCIETY OF MECHANICAL ENGINEERS for publication in the ASME JOURNAL OF APPLIED MECHANICS. Manuscript received by the ASME Applied Mechanics Division, May 23, 2001; final revision, Dec. 3, 2002. Associate Editor: N. C. Perkins. Discussion on the paper should be addressed to the Editor, Prof. Robert M. McMeeking, Department of Mechanical and Environmental Engineering University of California-Santa Barbara, Santa Barbara, CA 93106-5070, and will be accepted until four months after final publication of the paper itself in the ASME JOURNAL OF APPLIED MECHANICS. namics, which leads to a variational equation in terms of virtual displacements. However, due to the special character of the impulsive motion that the position of each particle is held fixed, the variation on the position such as the virtual displacement seems to be a little unnatural. On the other hand, in 1909, Jourdain [22] introduced the notion of variation on the velocity while keeping the time and the position fixed. This concept leads to the so-called Jourdain's variational equation, [22], or the principle of virtual power [23-26] for dynamical problems, and the corresponding variational equation on the (finite or infinitesimal) velocity variations is termed the second form of fundamental equations in [3]. Since the position is not changed for such variations, it is deemed that the fundamental equation in velocity variations is more appropriate to be used for dealing with constrained impulsive problems, cf. [3,11]. However, in the application of the variational equation, it is required to clearly identify the associated conditions on the velocity variations. For the aforementioned different types of impulsive constraints, we thus have to indicate their relations with the compatibility conditions on the velocity variations in the basic principle.

In this paper, we shall separate the impulsive motion of the system into three phases. The prior motion and the posterior motion refer to the motion immediately before and after the instant at which the impulsive motion occurs, respectively. In addition to the regular constraints, the system is subject to live and released constraints in the prior motion, while it is restricted by live and inert constraints in the posterior motion. To accommodate the elastic constraints, which are imposed on the system during the impulsive motion, it is postulated that the system is in the state of the third phase, i.e., the so-called virtual motion, in which live, inert, and elastic constraints are active. We note that the compatibility conditions on the velocity variations induced from the kinematic conditions in the virtual motion are those should be included in the fundamental variational equation. Accordingly, analogous to the principle of virtual power for finite-force motion, we propose the principle of velocity variations for the constrained impulsive motion of finite degree-of-freedom system as enunciated in Section 4. This fundamental principle essentially says that the applied impulses can be divided into the effective impulses which generate the jumps of velocities, and the net applied impulses, which cannot change the jumps. Hence the sum total of the product of the latter and the velocity variations compatible with the constraints in the virtual motion must vanish.

From the fundamental principle, the basic variational equation for impulsive motion can be immediately derived. The phase of virtual motion may be described by generalized velocities, which are obtained from the regular geometric constraints, and quasi- 
velocities, which may arise naturally for a given problem. The holonomic or nonholonomic relationships between these velocities are solved to find a set of independent velocities, termed the virtual-privileged velocities, with total number being equal to the degree-of-freedom of the virtual motion. The variation of the velocity of each particle in the system can be then expressed in terms of the variations of the virtual-privileged velocities. Substituting the expressions into the basic variational equation, the socalled privileged impulse-momentum equations for impulsive motion are deduced, from which it is observed that the differences between the prior virtual-privileged momenta and the posterior virtual-privileged momenta are balanced by the applied virtualprivileged impulses. The privileged impulse-momentum equations, formulated in the virtual motion, can be then used to find the relation between the prior motion and the posterior motion. In particular, the conservation law of the virtual-privileged momenta can be stated for the constrained impulsive problem if there is no applied impulse. However, if elastic constraints appear, the privileged impulse-momentum equations are insufficient to determine the states in the posterior motion from the prior motion, and additional criteria, such as the law of impact, should be invoked.

While the privileged impulse-momentum equations for impulsive motion subject to regular constraints derived here are similar to those obtained by using Kane's approach, [7,27], the impulsive constraints and the notion of virtual motion were not discussed there. The basic variational equation introduced here provides the more suitable scenario to deal with the impulsive constraints, and paves the way for further synthesis with other methodologies for either continuous motion or for body with infinite degree-offreedom. The privileged velocities mentioned before, called the generalized speeds in [7], are systematically obtained here by using the active kinematic constraints and the expressions of the quasi-velocities. For some problems associated with complex mechanical systems, Kane's approach may be superior to the others in deriving the equations of motion for finite-force problems, cf. [28]. The methodology proposed in this paper thus also have those advantages due to the similarity.

On the other hand, from the geometric point of view, the privileged equations may be viewed as the balance of the projections of the underlying quantities to some appropriate subspaces determined by the constraints, cf. [20,29-31]. In modern geometric mechanics, [32,33], the virtual displacement is regarded as the tangent vectors to the configuration space and the geometric formulation of Lagrangian mechanics, [34,35], is mainly based on the D'Alembert principle. Since the velocity variations are essentially the tangent vectors to the velocity space, it may be interesting to develop the geometric formulation for the principle of virtual power or the principle of velocity variations. In particular, the treatments of the force as a 1-form, [35], a horizontal 1-form, [34], or a semibasic 1-form, [12], in geometric Lagrangian mechanics may not be appropriate in formulating the Jourdain variational equation in modern geometric terminologies.

The rest of this paper is organized as follows. The classification of impulsive constraints and the separation of different phases of motion are discussed in Section 2. The kinematic constraints for various phases of motion and the notion of the privileged velocities are described in Section 3. The fundamental principle for impulsive motion is then stated in Section 4, along with the derivation of the privileged impulse-momentum equations for impulsive motion. The application of the equations to solve the priorvalue problems is discussed in Section 5. Section 6 describes the application of the fundamental principle and the privileged equations to a system including rigid bodies. A physical example, i.e., the sleigh under impulsive motion, is then given in Section 7 to illustrate the proposed methodology, in which Newton's method and Lagrange's method are also used to solve the same problem to manifest their differences. Some concluding remarks are finally given in Section 8.

\section{Classification of Impulsive Motions}

The impulsive motion of a mechanical system refers to a sudden change of motion due to either applied impulses or sudden changes of constraints. The limiting case of a large force acting for a short interval of time may be viewed as an impulsive force, and an impulse is defined to be the limit of the integration of an impulsive force over the short interval. If sudden changes of constraints occur, each particle in the system may be treated as being imposed by the impulsive constraint forces, or constraint impulses. Similar to the notion of constraint force for a nonimpulsive motion, or termed finite-force motion, the constraint impulses only appear when the constraints exist. During the impulsive motion, each particle in the system may be impressed by external impulses or the interactive impulses from the other particles. The total impulses may be further grouped into the applied impulses and the constraint impulses. The problem associated with the impulsive motion of a constrained mechanical system exerted by applied impulses is termed the constrained impulsive problem. The kinematic condition that restricts the motion in the whole process of the impulsive motion is called a regular constraint. On the other hand, the constraint that appears or vanishes during the impulsive motion is named the impulsive constraint. Either regular constraints or impulsive constraints may exert constraint impulses during the impulsive motion.

To illustrate these notions, we consider a ball rolling on a rough surface. The ball rolls without sliding and thus is subject to some nonholonomic constraints. If there is an external impulse acting on the ball while the condition of pure rolling is not affected, these nonholonomic constraints are regular. If the ball hits the wall such that there are new constraints appearing, such constraints are the impulsive constraints. According to the duration of their effectiveness, the impulsive constraints may be further classified into the following categories:

\section{(a) Inert Impulsive Constraints}

For an inelastic ball falling on the floor, the constraint occurs during the impulsive motion, and holds after the motion. Such impulsive constraints that appear during the impulsive motion and are satisfied afterwards are called the inert impulsive constraints.

\section{(b) Elastic Impulsive Constraints}

If the ball is elastic and is dropped to the floor, the sudden constraints imposed by the floor during the impulsive motion disappear afterwards. Such impulsive constraints are termed the elastic impulsive constraints.

\section{(c) Live Impulsive Constraints}

If a ball rolls on a rough surface, and the surface is suddenly set to move, the form of the original constraints still holds, but some terms may be changed due to the sudden motion of the surface. As an example, consider a vertical disk with radius $a$ rolling on a plane moving with velocity $\left(\dot{x}_{p}, \dot{y}_{p}\right)$. The rolling-without-sliding constraints can be expressed as

$$
\begin{aligned}
& \dot{x}_{c}-a \dot{\phi} \cos \theta=\dot{x}_{p}, \\
& \dot{y}_{c}-a \dot{\phi} \sin \theta=\dot{y}_{p},
\end{aligned}
$$

where $\left(\dot{x}_{c}, \dot{y}_{c}\right)$ denotes the velocity of the center of the disk, and $\dot{\phi}, \theta$ represent the spin rate, the heading angle of the disk, respectively. If the plane is moved suddenly so that $\dot{x}_{p}$ and $\dot{y}_{p}$ have abrupt changes, the set of constraints (1), (2) are varied accordingly. Such impulsive constraints are grouped as the live impulsive constraints.

\section{(d) Released Impulsive Constraints}

In contrast to the inert impulsive constraints, there may be some constraints vanishing during the impulsive motion and afterwards. Such impulsive constraints are termed the released impulsive constraints. 


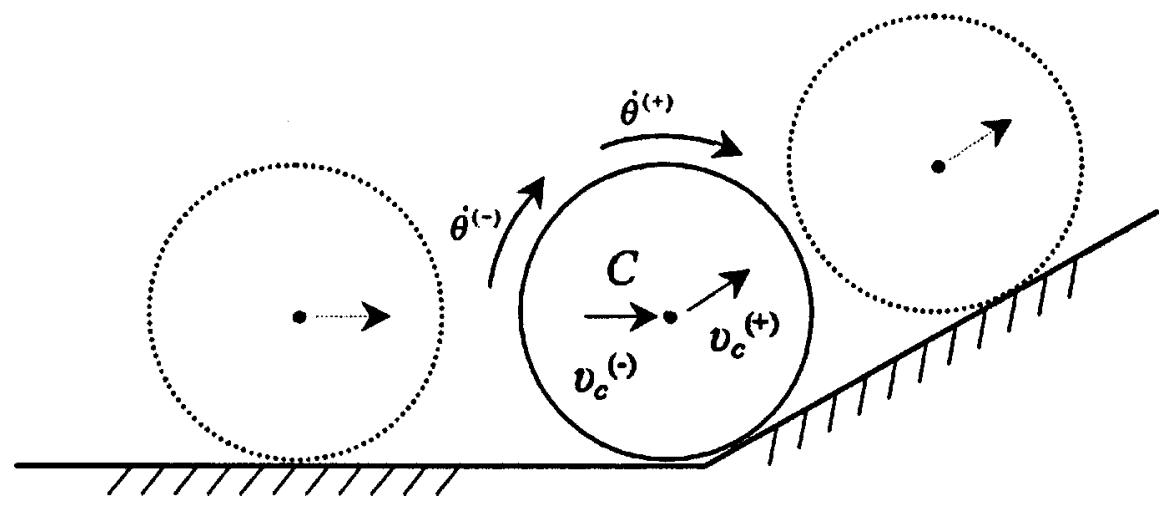

Fig. 1 A ball rolls across the boundary between two surfaces

Although a live impulsive constraint may be divided into a combination of a released constraint and an inert constraint, with certain specific relationship, it is treated specially in one category due to its frequent appearance and the distinct structure, [3].

A similar scheme for the classification of the constraints occurring in the impulsive motion was given by Appell [1], in which among the four types there the first one is essentially the aforementioned regular constraints, the second type is the inert constraints, the fourth type corresponds to the elastic constraints, while the live constraints defined above is not mentioned. In contrast to the released constraint, Appell's third type of constraints is regarded active during the impulsive motion. However, from some observations, the notion of released constraints introduced here may be more appropriate to be adopted. Consider a ball being grasped in hand, and suddenly released at $\bar{t}$. It is obvious that the velocity of the ball will not change during the short interval of the impulsive motion and immediately after the instant $\bar{t}$. The constraint of grasping is suddenly released and is thus not active during the impulsive motion.

Although the system undergoing released constraints will not experience a sudden change of velocity, the degree-of-freedom of its motion varies significantly. In fact, released constraints often occur simultaneously along with other kinds of impulsive constraints or the imposition of applied impulses to result in impulsive motion. Consider a ball rolling on a rough horizontal surface, and suddenly bumping into an inclined rough surface such that it may start to ascend, cf. Fig. 1. At the instant of impact, the constraint from the horizontal surface is released, which should not provide any action (constraint impulse) on the ball except that the degree-of-freedom of the system is increased. The suddenly appeared constraint from the inclined surface, the inert constraint, can then be imposed on the motion.

The difference between the released constraints and Appell's third type of constraints affects the analysis of impulsive motion. In fact, in [8], the constrained impulsive problems associated with the Appell's first and the second types of constraints, termed persistent constraints, are described to be determinant, while for the third and the fourth type, the problems are claimed to be indeterminant, for which additional laws are required. From the observations made above, it is seen that the problems associated the released constraint are determinant, and only problems with elastic constraint need more special treatment. However, it is noted that in the above-mentioned examples, the normal relative velocity between bodies before the impulsive motion subject to the released constraints vanishes. If it is nonzero, the corresponding impulsive constraint should be treated as a combination of a released constraint and an elastic one.

From the above discussions, the analysis of impulsive motion should take into account the occurrences of the impulsive constraints. Different classes of constraints may be active in different stages during the impulsive motion. This observation matches with the discussion in [12], where the analysis of motion subject to impulsive constraints based on modern geometric mechanics is performed individually for different types, i.e., permanent nonholonomic (regular), permanent impulsive (inert), elastic, etc.

To specifically identify the action of various impulsive constraints, we shall divide the impulsive motion into three phases. The motion immediately before the instant $\bar{t}$ at which the impulsive motion occurs is called the prior motion, during which live and released constraints are active. Immediately after $\bar{t}$, the system is in the posterior motion and is subject to inert and live constraints. In between the prior motion and the posterior motion, the system is influenced by live, inert, and elastic constraints, and may be postulated in the so-called virtual motion. The classification of the impulsive constraints and the separation of three phases during the impulsive motion pave the way to systematically treat the constrained impulsive problems in the following sections.

\section{Kinematics of Impulsive Motions}

Consider a system $\mathcal{S}$ of particles, whose number may be finite or infinite, indexed by an index set $I$. Each particle $P_{i}$ in $\mathcal{S}, i$ $\in I$, with mass $m_{i}$, is located at the position $\mathbf{r}_{i} \in \mathbb{R}^{3}$. The configuration of $\mathcal{S}$ is specified by the positions of all particles in $\mathcal{S}$. The system is assumed to be of finite degree-of-freedom, in the sense that there is a set of $N$ particles in $\mathcal{S}$ such that the positions of all particles can be determined from those of the $N$ particles, i.e.,

$$
\mathbf{r}_{i}=\mathbf{r}_{i}\left(x_{1}, \cdots, x_{3 N}, t\right), \quad i \in I,
$$

where $\left(x_{3 j-2}, x_{3 j-1}, x_{3 j}\right)$ are the three components of $\mathbf{r}_{j}, j$ $=1, \cdots, N$. A system consisting of particles of finite number or rigid continua is in such a category. Although the methodology developed in this paper may be extended formally to systems with infinite degree-of-freedom, the finite degree-of-freedom is assumed here to avoid some technical difficulties and to enhance the clarity of later discussions.

If $\mathcal{S}$ is exerted by applied impulses or undergoes sudden change of constraints at instant $\bar{t}$, the system may experience a sudden change of motion between $(\bar{t}-, \bar{t}+)$, i.e., the interval immediately before and after the impulsive motion. Although it is more physically realistic to regard the duration of the motion in a very short interval $[\bar{t}-\tau, \bar{t}+\tau]$, the analysis of motion of $\mathcal{S}$ is usually performed by taking the limit as $\tau \rightarrow 0$. For each particle $P_{i}$ in $\mathcal{S}$, its velocity, which is finite during the motion, may have a discontinuity at $\bar{t}$, with the corresponding jump of velocity $\Delta \mathbf{v}_{i}=\mathbf{v}_{i}^{(+)}$ $-\mathbf{v}_{i}^{(-)}$, where $\mathbf{v}_{i}^{(-)}$(prior velocity) and $\mathbf{v}_{i}^{(+)}$(posterior velocity) denote the velocities of $P_{i}$ at $\bar{t}-$ and $\bar{t}+$, respectively. The integration of the velocity $\dot{\mathbf{r}}_{i}(t)$ with respect to time over the interval $[\bar{t}-\tau, \bar{t}+\tau]$ is seen to be zero as $\tau \rightarrow 0$, due to the fact that the velocity is finite in the interval. Accordingly, the position of each particle in $\mathcal{S}$ is unchanged during the impulsive motion, denoted 
by $\overline{\mathbf{r}}_{i}, i \in I$, and only the jump of velocity may occur. The above reasoning can be formalized by using the Dirac theory of distributions as in [14] to show that the position is fixed during the impulsive motion, which is essentially an a priori assumption in [9].

Let the system be subject to independent regular constraints including $K$ geometric constraints and $L$ linear kinematic constraints. Due to the assumption of finite degree-of-freedom, the constraints on every particles in $\mathcal{S}$ can be transformed into the constraints on the selected $N$ particles. The regular constraints may be then expressed as

$$
\begin{gathered}
f_{s}\left(x_{1}, \cdots, x_{3 N}, t\right)=0, \quad s=1, \cdots, K \\
\sum_{i=1}^{3 N} A_{s i}\left(x_{1}, \cdots, x_{3 N}, t\right) \dot{x}_{i}+a_{s}\left(x_{1}, \cdots, x_{3 N}, t\right)=0, \quad s=1, \cdots, L
\end{gathered}
$$

respectively. By solving the $K$ geometric constraints, the position of each particle can be expressed parametrically by introducing $n(=3 N-K)$ number of generalized coordinates $q_{1}, \cdots, q_{n}$, as

$$
\mathbf{r}_{i}=\hat{\mathbf{r}}_{i}(\mathbf{q}, t), \quad i \in I,
$$

where $\mathbf{q}$ denotes the ensemble $\left(q_{1}, \cdots, q_{n}\right)$. Differentiating $\mathbf{r}_{i}$ with respect to time, the velocities can be then written as

$$
\dot{\mathbf{r}}_{i}=\sum_{j=1}^{n} \frac{\partial \hat{\mathbf{r}}_{i}}{\partial q_{j}} \dot{q}_{j}+\frac{\partial \hat{\mathbf{r}}_{i}}{\partial t}, \quad i \in I,
$$

where $\dot{q}_{j}=d q_{j} / d t, j=1, \cdots, n$, are the generalized velocities of the system. Substituting the components of the velocities of the $N$ particles in Eq. (5) into the linear kinematic constraints (4), the constraints on the generalized velocities can be expressed as

$$
\sum_{j=1}^{n} B_{s j}^{R}(\mathbf{q}, t) \dot{q}_{j}+b_{s}^{R}(\mathbf{q}, t)=0, \quad s=1, \cdots, L .
$$

For a constrained mechanical system, it is sometimes convenient to describe the motion in terms of $p$ quasi-velocities $\dot{\pi}_{k}$, defined by

$$
\dot{\pi}_{k} \triangleq \sum_{j=1}^{n} C_{k j}(\mathbf{q}, t) \dot{q}_{j}+c_{k}(\mathbf{q}, t), \quad k=1, \cdots, p,
$$

in which the right-hand side is nonintegrable. Since the regular constraints are imposed during the whole process, the motion of the system in all three phases can be then characterized by the combined set of $n$ generalized velocities and $p$ quasivelocities. The configuration at $\bar{t}$ is specified by the generalized coordinates $\bar{q}_{1}, \cdots, \bar{q}_{n}$, and the prior motion, the posterior motion are described by the combined sets of velocities $\quad\left(\dot{q}_{1}^{(-)}, \cdots, \dot{q}_{n}^{(-)}, \dot{\pi}_{1}^{(-)}, \cdots, \dot{\pi}_{p}^{(-)}\right), \quad$ and $\quad\left(\dot{q}_{1}^{(+)}, \cdots, \dot{q}_{n}^{(+)}\right.$, $\left.\dot{\pi}_{1}^{(+)}, \cdots, \dot{\pi}_{p}^{(+)}\right)$, respectively.

Other than the regular constraints, additional constraints may be imposed on or released from $\mathcal{S}$ during the impulsive motion, which may include geometric ones. For example, consider the motion of two rigid balls rolling on a plane and colliding with each other. At the instant of collision, there is an impulsive geometric constraint that the distance between two centers equals the sum of their radii. However, unlike the regular geometric constraints, the impulsive geometric constraints cannot be used to reduce the number of generalized coordinates, since they are only satisfied at the instant of impulsive motion. A configuration for the constrained impulsive motion is said to be possible if these impulsive geometric constraints are satisfied at the instant $\bar{t}$, which may be described by $\left(\bar{q}_{1}, \cdots, \bar{q}_{n}\right)$ that satisfies the impulsive geometric constraints. After determining the possible configuration at the instant of impulsive motion, these impulsive geometric constraints should be transformed into linear kinematic forms. Com- bining with the others, it is assumed that there are totally $L_{I}$ impulsive linear kinematic constraints in the form of

$$
\sum_{j=1}^{n} B_{s j}^{I}(\mathbf{q}, t) \dot{q}_{j}+b_{s}^{I}(\mathbf{q}, t)=0, \quad s=1, \cdots, L_{I} .
$$

According to the classification in the previous section, these impulsive constraints are further divided into four groups, i.e., live, inert, elastic, and released, with numbers of $l_{\mathcal{L}}, l_{\mathcal{I}}, l_{\mathcal{E}}, l_{\mathcal{R}}$, respectively. For each group, the corresponding terms in the form of Eq. (8) will be denoted by $\left(B_{s j}^{\mathcal{L}}, b_{s}^{\mathcal{L}}\right),\left(B_{s j}^{\mathcal{I}}, b_{s}^{\mathcal{I}}\right),\left(B_{s j}^{\mathcal{E}}, b_{s}^{\mathcal{E}}\right)$, and $\left(B_{s j}^{\mathcal{R}}, b_{s}^{\mathcal{R}}\right)$, respectively, in which the inhomogeneous terms of the live constraints, $b_{s}^{\mathcal{L}}, s=1, \cdots, l_{\mathcal{L}}$, are different before and after $\bar{t}$. As discussed before, the prior motion satisfies live and released constraints, the posterior motion satisfies live and inert constraints, while live, inert, and elastic constraints are active in the virtual motion. For each phase of the impulsive motion, the impulsive constraints and the regular constraints (6) are combined into a set of active kinematic conditions on the motion. The corresponding degrees-of-freedom of the system are thus $m^{b}=n-L$ $-l_{\mathcal{L}}-l_{\mathcal{R}}, m^{a}=n-L-l_{\mathcal{L}}-l_{\mathcal{I}}$, and $m^{\prime}=n-L-l_{\mathcal{L}}-l_{\mathcal{I}}-l_{\mathcal{E}}$ for prior motion, posterior motion, and virtual motion, respectively. Here, and in what follows, the superscripts " $b$," " $a$," and " ", are used to indicate the validation of the corresponding terms for $t$ $<\bar{t}-, t>\bar{t}+$, and $t \in(\bar{t}-, \bar{t}+)$, respectively.

Recall that the motion is described by the aggregate of $n$ generalized velocities and $p$ quasi-velocities, which are related by different sets of constraints for the three phases. In particular, for the virtual motion, the generalized velocities and the quasivelocities satisfy the $L+l_{\mathcal{L}}+l_{\mathcal{I}}+l_{\mathcal{E}}$ equations of kinematic constraint and $p$ equations of quasi-velocities (7). By solving these equations, which are linear in velocities, we may select $m^{\prime}$ number of independent velocities, $\dot{\phi}_{1}^{\prime}, \cdots, \dot{\phi}_{m^{\prime}}^{\prime}$, called the virtualprivileged velocities, such that the generalized velocities and the quasi-velocities compatible with the kinematic conditions can be expressed as

$$
\begin{gathered}
\dot{q}_{j}=\sum_{\sigma=1}^{m^{\prime}} D_{j \sigma}^{\prime}(\mathbf{q}, t) \dot{\phi}_{\sigma}^{\prime}+d_{j}^{\prime}(\mathbf{q}, t), \quad j=1, \cdots, n, \\
\dot{\pi}_{k}=\sum_{\sigma=1}^{m^{\prime}} G_{k \sigma}^{\prime}(\mathbf{q}, t) \dot{\phi}_{\sigma}^{\prime}+g_{j}^{\prime}(\mathbf{q}, t), \quad k=1, \cdots, p .
\end{gathered}
$$

Furthermore, by substituting Eq. (9) into Eq. (5), one may express the possible velocity of each particle of $\mathcal{S}$ in virtual motion as

$$
\dot{\mathbf{r}}_{i}=\sum_{\sigma=1}^{m^{\prime}} \beta_{i \sigma}^{\prime}(\mathbf{q}, t) \dot{\phi}_{\sigma}^{\prime}+\gamma_{i}^{\prime}(\mathbf{q}, t), \quad i \in I,
$$

where

$$
\beta_{i \sigma}^{\prime}=\sum_{j=1}^{n} \frac{\partial \hat{\mathbf{r}}_{i}}{\partial q_{j}} D_{j \sigma}^{\prime}, \quad \gamma_{i}^{\prime}=\sum_{j=1}^{n} \frac{\partial \hat{\mathbf{r}}_{i}}{\partial q_{j}} d_{j}^{\prime}+\frac{\partial \hat{\mathbf{r}}_{i}}{\partial t},
$$

are continuously differentiable functions of $(\mathbf{q}, t)$, but not of $\dot{\mathbf{q}}$, since the kinematic constraints and the quasi-velocity equations are all linear in velocity.

Analogous to the process of selecting the virtual-privileged velocities, the set of prior-privileged velocities $\left\{\dot{\phi}_{\sigma}^{b}\right\}$, and that of posterior-privileged velocities $\left\{\dot{\phi}_{\sigma}^{a}\right\}$ can be obtained for the prior motion and the posterior motion, respectively. Similar forms of Eqs. (9), (10), (11) are found for each phase to describe the motion, with the corresponding notations summarized in Table 1. It is noted that the selection of privileged velocities basically depends on the coefficients of the velocity terms, i.e., $B_{s j}^{I}, B_{s j}^{R}$, and $C_{k j}$, and hence the discontinuity of the inhomogeneous terms arising from the live constraints does not affect the selection process. 
Table 1 Summary of the transformations

\begin{tabular}{lccc}
\hline \hline & Prior Motion & Virtual Motion & Posterior Motion \\
\hline $\begin{array}{l}\text { Active Impulsive } \\
\text { Constraints }\end{array}$ & Released, Live & Live, Elastic, Inert & Live, Inert \\
Privileged & $\phi_{\sigma}^{b}, \sigma=1, \ldots, m^{b}$ & $\phi_{\sigma}^{\prime}, \sigma=1, \ldots, m^{\prime}$ & $\phi_{\sigma}^{a}, \sigma=1, \ldots, m^{a}$ \\
Coordinates & $\left(D_{j \sigma}^{b}, d_{j}^{b}\right)$ & $\left(D_{j \sigma}^{\prime}, d_{j}^{\prime}\right)$ & $\left(D_{j \sigma}^{a}, d_{j}^{a}\right)$ \\
$\dot{\phi}_{\sigma}$ to $\dot{q}_{j}(9)$ & $\left(G_{k \sigma}^{b}, g_{k}^{b}\right)$ & $\left(G_{k \sigma}^{\prime}, g_{k}^{\prime}\right)$ & $\left(G_{k \sigma}^{a}, g_{k}^{a}\right)$ \\
$\dot{\phi}_{\sigma}$ to $\dot{\pi}_{k}(10)$ & $\left(\beta_{i \sigma}^{b}, \gamma_{i}^{b}\right)$ & $\left(\beta_{i \sigma}^{\prime}, \gamma_{i}^{\prime}\right)$ & $\left(\beta_{i \sigma}^{a}, \gamma_{i}^{a}\right)$ \\
$\dot{\phi}_{\sigma}$ to $\dot{\mathbf{r}}_{i}(11)$ & & & \\
\hline \hline
\end{tabular}

If all the kinematic constraints are regular, i.e., there is no impulsive constraint, the kinematic conditions for the three phases of motion are the same, with the same degree-of-freedom $m^{\prime}=m^{b}$ $=m^{a}=m=n-L$. The same set of privileged velocities may be selected as well to describe the motion for three phases, i.e., $\dot{\phi}_{\sigma}^{\prime}$ $=\dot{\phi}_{\sigma}^{b}=\dot{\phi}_{\sigma}^{a}=\dot{\phi}_{\sigma}, \sigma=1, \cdots, m$. The problem is then simplified to the classical problem of impulsive motion, and the method presented in this paper is similar to the one used in [7].

An impulsive problem that the prior motion is given and the posterior motion is to be determined is called a prior-value problem (analogous to the initial-value problem for differential equations). For such problems, the posterior velocity of each particle $P_{i}$ in $\mathcal{S}$ can be expressed in terms of the posterior-privileged velocities evaluated at $\bar{t}+$ as

$$
\mathbf{v}_{i}^{(+)}=\sum_{\sigma=1}^{m^{a}} \beta_{i \sigma}^{a}(\overline{\mathbf{q}}, \bar{t}) \dot{\phi}_{\sigma}^{a(+)}+\gamma_{i}^{a}(\bar{t}+), \quad i \in I .
$$

From the previous equations, once the posterior-privileged velocities are determined, the posterior velocity of each particle in $\mathcal{S}$ can be obtained subsequently.

To solve the prior-value problem, fundamental principles in mechanics needs to be invoked. To deal with the systems subject to constraints for finite-force problems, many variational principles have been developed, in which the concept of variations is introduced to "test" the limitations from the constraints. These variations are essentially infinitesimal quantities imposed on the system variables. As reviewed in [25], there are basically three types of variations. In the principle of virtual work or D'Alembert's principle in dynamics, the virtual displacement is an infinitesimal and instantaneous displacement imposed on the configuration of the system. In the principle of least constraint or the Gauss principle, [36], the variation is imposed on the acceleration of each particle while keeping the time, the position, and the velocity unchanged. On the other hand, in Jourdain's principle, [22,23], or the principle of virtual power, $[24,26]$, the variation is on the velocity, denoted by $\delta_{1} \mathbf{v}_{i}$, which is instantaneous $\left(\delta_{1} t=0\right)$ and stationary $\left(\delta_{1} \mathbf{r}_{i}\right.$ $=0)$.

In the literature, the term of virtual displacement and that of virtual velocity are sometimes used interchangeably. This may be due to the reason that in the original work of Bernoulli [37], the principle of virtual work is called the principle of virtual velocity. To tell the difference between the virtual displacement and the variation of velocity defined above, the latter shall be termed the velocity variation in this paper. In modern geometric mechanics, the virtual displacement is viewed as a tangent vector to the configuration space, [38], due to its infinitesimal character. Analogously, the velocity variation may be treated as a tangent vector to the velocity space, and is thus intrinsically different from the notion of virtual displacement.

The velocity variation plays a central role in the fundamental principle for impulsive motion presented in the next section, which requires that they must be compatible with the kinematic conditions of constraints in the virtual motion. From the above discussions, any possible velocity of each particle in virtual motion can be represented in terms of the virtual-privileged veloci- ties, Eq. (11). By taking $\delta_{1}$-variation on (11), the velocity variation $\delta_{1} \dot{\mathbf{r}}_{i}$ can be then expressed in terms of the variations of the virtual-privileged velocities $\delta_{1} \dot{\phi}_{\sigma}^{\prime}$ as

$$
\delta_{1} \dot{\mathbf{r}}_{i}=\sum_{\sigma=1}^{m^{\prime}} \beta_{i \sigma}^{\prime}(\mathbf{q}, t) \delta_{1} \dot{\phi}_{\sigma}^{\prime}, \quad i \in I .
$$

Since the virtual-privileged velocities $\dot{\phi}_{\sigma}^{\prime}$ are independent, there is no constraint on the corresponding variations $\delta_{1} \dot{\phi}_{\sigma}^{\prime}$. This fact shall be used in the next section to establish the required equations of jumps from the variational equation.

\section{Kinetic Equations for Impulsive Motions}

The dynamics of impulsive motion may be thought of as the limiting case for a finite-force problem. In addition to the D'Alembert-Lagrange equation and the Gauss-Gibbs equation, Jourdain in 1909, [22], established a variational equation for systems subject to linear kinematic constraints in terms of the infinitesimal variation of velocities. Later, Pars [3] extended the equations to accommodate the finite variations of velocities, and obtained the so-called second form of the fundamental equations as

$$
\sum_{i \in I}\left(m_{i} \ddot{\mathbf{r}}_{i}-\mathbf{F}_{i}^{(A)}\right) \cdot \Delta_{1} \dot{\mathbf{r}}_{i}=0,
$$

where $\ddot{\mathbf{r}}_{i}$ is the actual acceleration of particle $P_{i}, \mathbf{F}_{i}^{(A)}$ denotes the resultant applied force acting on $P_{i}$, and $\Delta_{1} \dot{\mathbf{r}}_{i}$ is an arbitrary finite or infinitesimal variation of possible velocity compatible with the constraints.

To extend the previous variational equation to deal with the constrained impulsive problem, due to the appearance of the impulsive constraints, the set of conditions from the constraints with which the velocity variations must be compatible should be identified. It may be envisioned that during the impulsive motion, the system undergoes the stage of virtual motion, during which the system satisfies the kinematic conditions from the regular constraints and the impulsive constraints including the types of inert elastic, and live. As a result, the velocity variations for virtual motion must be compatible with the conditions induced from these kinematic conditions. Substituting Eq. (13) into Eq. (14), and noting the independency of $\left\{\delta_{1} \dot{\phi}_{\sigma}^{\prime}\right\}_{\sigma=1, \cdots, m^{\prime}}$, we obtain

$$
\sum_{i \in I}\left(m_{i} \ddot{\mathbf{r}}_{i}-\mathbf{F}_{i}^{(A)}\right) \cdot \beta_{i \sigma}^{\prime}=0, \quad \sigma=1, \cdots, m^{\prime}
$$

where the acceleration of each particle $P_{i}$ may be very large during the impulsive motion, and leads to the jump of the corresponding velocity.

To obtain the relation between the prior motion and the posterior motion for the system $\mathcal{S}$, the previous equations are further expressed as 


$$
\begin{gathered}
\frac{d}{d t}\left(\sum_{i \in I} m_{i} \dot{\mathbf{r}}_{i} \cdot \beta_{i \sigma}^{\prime}\right)-\sum_{i \in I} m_{i} \dot{\mathbf{r}}_{i} \cdot\left(\sum_{j=1}^{n} \frac{\partial \beta_{i \sigma}^{\prime}}{\partial q_{j}} \dot{q}_{j}+\frac{\partial \beta_{i \sigma}^{\prime}}{\partial t}\right) \\
-\sum_{i \in I} \mathbf{F}_{i}^{(A)} \cdot \beta_{i \sigma}^{\prime}=0, \quad \sigma=1, \cdots, m^{\prime} .
\end{gathered}
$$

Since $\beta_{i \sigma}^{\prime}$ is continuous and the velocities, $\dot{\mathbf{r}}_{i}, i \in I$, and $\dot{q}_{j}, j$ $=1, \cdots, n$ are finite, the second term in the previous equation is finite for each $\sigma=1, \cdots, m^{\prime}$. Let the oth virtual-privileged momentum of $\mathcal{S}$ and the $\sigma$ th virtual-privileged applied force acting on $\mathcal{S}$ be defined as

$$
\begin{gathered}
\mathrm{L}_{\sigma} \triangleq \sum_{i \in I} m_{i} \dot{\mathbf{r}}_{i} \cdot \beta_{i \sigma}^{\prime}(\mathbf{q}, t), \\
\mathrm{F}_{\sigma}^{(A)} \triangleq \sum_{i \in I} \mathbf{F}_{i}^{(A)} \cdot \beta_{i \sigma}^{\prime}(\mathbf{q}, t),
\end{gathered}
$$

respectively. Integrating Eq. (15) from $\bar{t}-\tau$ to $\bar{t}+\tau$, and letting $\tau \rightarrow 0$, the second term vanishes, and the privileged impulsemomentum equations can be established:

$$
\mathrm{L}_{\sigma}^{(+)}-\mathrm{L}_{\sigma}^{(-)}=\mathrm{P}_{\sigma}^{(A)}, \quad \sigma=1, \cdots, m^{\prime},
$$

where

$$
\begin{aligned}
& \mathrm{L}_{\sigma}^{(-)} \triangleq \sum_{i \in I} m_{i} \mathbf{v}_{i}^{(-)} \cdot \beta_{i \sigma}^{\prime}(\overline{\mathbf{q}}, \bar{t}), \\
& \mathrm{L}_{\sigma}^{(+)} \triangleq \sum_{i \in I} m_{i} \mathbf{v}_{i}^{(+)} \cdot \beta_{i \sigma}^{\prime}(\overline{\mathbf{q}}, \bar{t}),
\end{aligned}
$$

denote the oth prior and posterior virtual-privileged momenta, respectively, and

$$
\mathrm{P}_{\sigma}^{(A)} \triangleq \int_{\bar{t}-}^{\bar{t}+} \mathrm{F}_{\sigma}^{(A)} d t=\int_{\bar{t}-}^{-} \sum_{i \in I} \mathbf{F}_{i}^{(A)} \cdot \beta_{i \sigma}^{\prime}(\mathbf{q}, t) d t
$$

is the oth virtual-privileged applied impulse.

Again, by the continuity of $\beta_{i \sigma}^{\prime}$ and the negligible change of configuration and interval of time during the impulsive motion, the virtual-privileged applied impulses can be further expressed as

$$
\begin{gathered}
\mathrm{P}_{\sigma}^{(A)}=\sum_{i \in I}\left(\int_{\bar{t}-}^{\bar{t}+} \mathbf{F}_{i}^{(A)} d t\right) \cdot \beta_{i \sigma}^{\prime}(\overline{\mathbf{q}}, \bar{t})=\sum_{i \in I} \mathbf{P}_{i}^{(A)} \cdot \bar{\beta}_{i \sigma}^{\prime}, \\
\sigma=1, \cdots, m^{\prime},
\end{gathered}
$$

where $\mathbf{P}_{i}^{(A)} \triangleq \int_{\bar{t}-}^{\bar{t}+} \mathbf{F}_{i}^{(A)} d t$ is the applied impulse acting on particle $P_{i}, i \in I$. Substituting the previous equation into (18), the privileged impulse-momentum equations are rewritten as

$$
\sum_{i \in I}\left(m_{i} \mathbf{v}_{i}^{(+)}-m_{i} \mathbf{v}_{i}^{(-)}-\mathbf{P}_{i}^{(A)}\right) \cdot \beta_{i \sigma}^{\prime}(\overline{\mathbf{q}}, \bar{t})=0, \quad \sigma=1, \cdots, m^{\prime} .
$$

From Eq. (22), given applied impulses acting on $\mathcal{S}$, the virtualprivileged applied impulses can be determined, and the jumps of the $m^{\prime}$ virtual-privileged momenta defined as

$$
\Delta \mathrm{L}_{\sigma} \triangleq \mathrm{L}_{\sigma}^{(+)}-\mathrm{L}_{\sigma}^{(-)}, \quad \sigma=1, \cdots, m^{\prime},
$$

are then obtained from Eq. (23).

The above derivation of the privileged impulse-momentum equations is based on the framework for finite-force problems. To gain more direct insight on the impulsive motion subject to constraints, and to avoid the technicalities in the transition from finite-force problems to impulsive problems, one may follow the principle of velocity variations for constrained impulsive motion as stated below:

Consider a system of particles $P_{i}, i \in I$, connected with one another in any way. The system may be subjected to applied im- pulses $\mathbf{P}_{i}^{(A)}$ on particle $P_{i}$. These applied impulses would impart to the free particles certain determinate jumps on their velocities. However, due to the constraints, regular or impulsive, the actual jumps are different from those on the free motions. Conceive that the applied impulse $\mathbf{P}_{i}^{(A)}$ be resolved into the effective impulse $\mathbf{P}_{i}^{(E)}=m_{i} \mathbf{v}_{i}^{(+)}-m_{i} \mathbf{v}_{i}^{(-)}$, and another component termed the net applied impulse. Owing to the constraints, only the effective impulses generate the actual jumps. The net applied impulses are incapable to change the jumps during the impulsive motion, and the sum total of the product of the net applied impulses and the velocity variations compatible with the constraints during the virtual motion must vanish.

This principle is an analog of the principle of virtual power for finite-force motion stated in [26], and the statements are similar to the exposition of D'Alembert's principle by Mach [37]. It is noted that Appell in [1] enunciated a fundamental principle for impulsive motion analogous to D'Alembert's principle, and, based on which, established the general variational equation in terms of virtual displacements. However, as discussed before, a virtual displacement refers to a variation of the position of the particle, which is in fact not allowed during the impulsive motion. While the variational equation corresponds to the previous principle has similar characteristics as that derived by Appell, the notion of velocity variation while keeping position fixed is more acceptable for the impulsive motion.

Based on this fundamental principle, the corresponding variational equation for impulsive motion can be immediately expressed as

$$
\sum_{i \in I}\left(m_{i} \mathbf{v}_{i}^{(+)}-m_{i} \mathbf{v}_{i}^{(-)}-\mathbf{P}_{i}^{(A)}\right) \cdot \delta_{1} \dot{\mathbf{r}}_{i}=0,
$$

for all $\delta_{1} \dot{\mathbf{r}}_{i}$ compatible with the constraints specified during the virtual motion at $(\overline{\mathbf{q}}, \bar{t})$. Substituting (13) into the previous variational equation and noting the independency of the variations of the virtual-privileged velocities, the privileged impulsemomentum Eqs. (23) immediately follow. This process may be viewed as the projection from the space of $\delta_{1} \dot{\mathbf{r}}_{i}$ to that of $\delta_{1} \dot{\phi}_{\sigma}^{\prime}$ through Eq. (13), and is essentially the basic idea for the so-called projection method, $[29,30]$. Similar techniques were also used in $[12,31]$ to derive jump conditions from a variational equation similar to (25) in virtual displacement without applied impulse. There the projection is from the tangent bundle to the distribution that annihilates the constraint submanifold. Since the velocity variations should reside in the second tangent bundle, or the jet space of order 2 , the projection through $\beta_{i \sigma}^{\prime}$ should be regarded as different from those in the tangent bundle.

Moreover, it is noted that the projection in Eq. (13) does not depend on the velocity. This is due to the fact that only linear kinematic constraints are treated in this paper. If the system is subject to nonlinear ones in the form of $\psi\left(x_{1}, \cdots, x_{3 N}, \dot{x}_{1}, \cdots, \dot{x}_{3 N}, t\right)=0$, the projection or the transformation may depend on the velocities. For impulsive problems, the discontinuity of the velocity then renders the transformation indeterminant. In [12], the impulsive constraints are associated with Chetaev bundle, [39], of reaction forces from the constraints, linear or nonlinear. However, if the kinematic constraint is nonlinear, it is not clear how to compute the corresponding impulse of these Chetaev forces, which depends on the velocity in general. As a result, more understandings on the mechanism for the impulsive motion subject to nonlinear kinematic constraints are desired.

\section{Prior-Value Problems}

We are now ready to apply the privileged impulse-momentum equations to study constrained impulsive problems. In the absence of applied impulses, from (18), we immediately have the following conservation law for constrained impulsive motion: the virtual-privileged momenta of the system are conserved if there is no applied impulse. 
For the prior-value problem, the prior virtual-privileged momentum can be computed from the state of the prior motion through Eq. (19). The posterior virtual-privileged momentum is then determined from the privileged impulse-momentum Eq. (23). To find the posterior motion, the relation between the $m^{\prime}$ posterior virtual-privileged momenta and the $m^{a}$ posterior-privileged velocities is constructed by substituting (12) into (20),

$$
\begin{aligned}
\mathrm{L}_{\sigma^{\prime}}^{(+)} & =\sum_{\sigma=1}^{m^{a}}\left(\sum_{i \in I} m_{i} \bar{\beta}_{i \sigma^{\prime}}^{\prime} \cdot \bar{\beta}_{i \sigma}^{a}\right) \dot{\phi}_{\sigma}^{a}(\bar{t}+)+\sum_{i \in I} m_{i} \bar{\beta}_{i \sigma^{\prime}}^{\prime} \cdot \gamma_{i}^{a}(\bar{t}+) \\
& =\sum_{\sigma=1}^{m^{a}} \mathrm{M}_{\sigma^{\prime} \sigma} \dot{\phi}_{\sigma}^{a}(\bar{t}+)+\mathrm{L}_{\sigma^{\prime} 0}^{(+)}, \quad \sigma^{\prime}=1, \cdots, m^{\prime}
\end{aligned}
$$

where

$$
\mathrm{M}_{\sigma^{\prime} \sigma^{\prime}} \triangleq \sum_{i \in I} m_{i} \bar{\beta}_{i \sigma^{\prime}}^{\prime} \cdot \bar{\beta}_{i \sigma}^{a}, \quad \mathrm{~L}_{\sigma^{\prime} 0}^{(+)} \triangleq \sum_{i \in I} m_{i} \bar{\beta}_{i \sigma^{\prime}}^{\prime} \cdot \gamma_{i}^{a}(\bar{t}+) .
$$

Here, and in what follows, the overbar of a continuous quantity denotes its value at $(\overline{\mathbf{q}}, \bar{t})$, e.g., $\bar{\beta}_{i \sigma}^{a}=\beta_{i \sigma}^{a}(\overline{\mathbf{q}}, \bar{t})$.

The previous set of $m^{\prime}$ Eqs. (26) is to be solved for the $m^{a}$ $\left(=n-L-l_{\mathcal{L}}-l_{\mathcal{I}}\right)$ unknowns, $\dot{\phi}_{\sigma}^{a}(\bar{t}+), \sigma=1, \cdots, m^{a}$. The difference between the number of equations and that of unknowns is $l_{\mathcal{E}}$, which is the number of elastic constraints. As a result, it is not possible to determine the posterior motion without additional $l_{\mathcal{E}}$ conditions if elastic constraints exist. Such conditions may be obtained by invoking the law of impact, cf. $[2,19]$, or $[27,40]$ for more recent developments. For example, to determine the posterior motion of a ball being dropped to the floor, the coefficient of restitution $\kappa$ is needed to find the extend of rebound. The elastic constraint is $\dot{\mathbf{r}} \cdot \mathbf{n}=0$, where $\mathbf{n}$ is the normal vector corresponding the floor, and the posterior velocity is related to the prior velocity as

$$
\mathbf{v}^{(+)} \cdot \mathbf{n}=-\kappa \mathbf{v}^{(-)} \cdot \mathbf{n} .
$$

From the previous example, it may be conceived that for the elastic constraints, $\sum_{j=1}^{n} B_{s j}^{\mathcal{E}} \dot{q}_{j}+b_{s}^{\mathcal{E}}=0, s=1, \cdots, l_{\mathcal{E}}$, there are $l_{\mathcal{E}}$ generalized coefficients of restitution $\kappa_{s}$ to bear the relationship between the "approaching" constraint functions and the "leaving" constraint functions as

$$
\sum_{j=1}^{n} \bar{B}_{s j}^{\mathcal{E}} \dot{q}_{j}^{(+)}+\bar{b}_{s}^{\mathcal{E}}=-\kappa_{s}\left(\sum_{j=1}^{n} \bar{B}_{s j}^{\mathcal{E}} \dot{q}_{j}^{(-)}+\bar{b}_{s}^{\mathcal{E}}\right), \quad s=1, \cdots, l_{\mathcal{E}},
$$

where the coefficients and the inhomogeneous terms are assumed to be continuous. From the formula of the posterior generalized velocities, with coefficients listed in Table 1 , the previous equations are further expressed as

$$
\begin{aligned}
& \sum_{\sigma=1}^{m^{a}}\left(\sum_{j=1}^{n} \bar{B}_{s j}^{\mathcal{E}} \bar{D}_{j \sigma}^{a}\right) \dot{\phi}_{\sigma}^{a}(\bar{t}+) \\
& \quad=-\sum_{j=1}^{n} \bar{B}_{s j}^{\mathcal{E}}\left(d_{j}^{a}(\bar{t}+)+\kappa_{s} \dot{q}_{j}^{(-)}\right)-\left(1+\kappa_{s}\right) \bar{b}_{s}^{\mathcal{E}}, \quad s=1, \cdots, l_{\mathcal{E}} .
\end{aligned}
$$

Equations (26), (27) can be then combined to obtain the $m^{a}$ posterior-privileged velocities, as illustrated by the following simple example.

Example 1. Consider a particle of mass $m$ being tossed to the floor $(z=0)$ with prior velocity $v_{x}^{(-)} \mathbf{e}_{x}+v_{z}^{(-)} \mathbf{e}_{z}$. Assume that the surface of the floor is ideally smooth, and the coefficient of restitution of the contact is $\kappa$. It is desired to determine the posterior velocity of the particle.

The impulsive constraint provided by the floor is $\dot{z}=0$, which is an elastic constraint. Hence, the virtual motion can be expressed as $\dot{\mathbf{r}}=\dot{x} \mathbf{e}_{x}$, where $\dot{x}$ can be selected as the privileged velocity for the virtual motion, and the coefficient corresponding to $\dot{x}$ is $\bar{\beta}_{1}^{\prime}$ $=\mathbf{e}_{x}$. The prior and posterior virtual-privileged momentum can be found to be $\mathrm{L}_{1}^{(-)}=\left(m v_{x}^{(-)} \mathbf{e}_{x}+m v_{z}^{(-)} \mathbf{e}_{z}\right) \cdot \mathbf{e}_{x}=m v_{x}^{(-)}, \quad \mathrm{L}_{1}^{(+)}$ $=\left(m v_{x}^{(+)} \mathbf{e}_{x}+m v_{z}^{(+)} \mathbf{e}_{z}\right) \cdot \mathbf{e}_{x}=m v_{x}^{(+)}$, respectively. The conservation of the virtual-privileged momentum leads to $v_{x}^{(-)}=v_{x}^{(+)}$. However, this equation is not sufficient to determine all the posterior velocities. The additional condition from the law of impact, i.e., $v_{z}^{(+)}=-\kappa v_{z}^{(-)}$, is needed to obtain the posterior velocity $v_{x}^{(-)} \mathbf{e}_{x}-\kappa v_{z}^{(-)} \mathbf{e}_{z}$.

In contrast to the previous case for an impulsive motion without elastic constraint, we have the same number of unknowns and equations in (26), and the coefficients $\bar{\beta}_{i \sigma}^{a}=\bar{\beta}_{i \sigma}^{\prime}$ if the same set of virtual-privileged velocities and posterior-privileged velocities is chosen. Accordingly, the matrix $\mathrm{M}$ formed by the components

$$
\mathrm{M}_{\sigma^{\prime} \sigma}=\sum_{i \in I} m_{i} \bar{\beta}_{i \sigma^{\prime}}^{\prime} \cdot \bar{\beta}_{i \sigma}^{\prime}, \quad \sigma, \sigma^{\prime}=1, \cdots, m^{\prime}
$$

is positive definite and shall be termed the privileged mass matrix. Under this framework, the posterior-privileged velocities can be obtained from the following equations:

$$
\dot{\phi}_{\sigma}^{a(+)}=\sum_{\sigma^{\prime}=1}^{m^{\prime}}\left(\mathrm{M}^{-1}\right)_{\sigma \sigma^{\prime}}\left(\mathrm{L}_{\sigma^{\prime}}^{(+)}-\mathrm{L}_{\sigma^{\prime} 0}^{(+)}\right), \quad \sigma=1, \cdots, m^{\prime},
$$

from which the posterior velocities can be determined from (12).

For the treatment of released constraints, the above deduction of determinacy is apparently different from that claimed in [8], which regards the third type of impulsive problem in [1] as indeterminant. To illustrate the difference, we consider again the problem of releasing a ball from hand grasping. As described in Section 2, the constraint of grasping is treated as a released constraint, which is effective before but ineffective during and after the impulsive motion. Since there is no kinematic constraint imposed in the virtual motion, the virtual-privileged velocities of the ball can be selected to be the three components of the linear velocity of the ball, and the corresponding virtual-privileged momenta are nothing but the three components of the linear momentum $M \mathbf{v}$. If hand does not provide additional applied impulse, from the conservation law of virtual-privileged momenta, we have $M \mathbf{v}^{(-)}=M \mathbf{v}^{(+)}$ $=0$ if the ball is released from rest. The posterior motion is then determined, and the motion of the ball bears no sudden change during the impulsive motion, only experiences sudden increase of the degree-of-freedom. From this simple example, it is seen that the notion of released constraint introduced in this paper is more appropriate to be adopted to treat the released constraints from grasping.

Now we consider the special case that there is neither inert constraints nor released constraints existing, while some live constraints appear. For such impulsive motion, as discussed in the previous section, the same set of privileged velocities may be chosen for the prior motion and the posterior motion, $\dot{\phi}_{\sigma}^{b}=\dot{\phi}_{\sigma}^{a}$, where $\sigma=1, \cdots, m^{a}\left(=m^{b}=n-L-l_{\mathcal{L}}\right)$, and we have

$$
\begin{gathered}
\mathbf{v}_{i}^{(-)}=\sum_{\sigma=1}^{m^{a}} \bar{\beta}_{i \sigma}^{a} \dot{\phi}_{\sigma}^{a(-)}+\gamma_{i}^{a}(\bar{t}-), \\
\mathbf{v}_{i}^{(+)}=\sum_{\sigma=1}^{m^{a}} \bar{\beta}_{i \sigma}^{a} \dot{\phi}_{\sigma}^{a(+)}+\gamma_{i}^{a}(\bar{t}+), \quad i \in I .
\end{gathered}
$$

The privileged impulse-momentum Eqs. (23) then implies that

$$
\sum_{\sigma=1}^{m^{a}} \mathrm{M}_{\sigma^{\prime} \sigma} \Delta \dot{\phi}_{\sigma}+\Delta \mathrm{L}_{\sigma^{\prime} 0}-\mathrm{P}_{\sigma^{\prime}}^{(A)}=0, \quad \sigma^{\prime}=1, \cdots, m^{\prime},
$$


where $\Delta \dot{\phi}_{\sigma}=\dot{\phi}_{\sigma}^{a(+)}-\dot{\phi}_{\sigma}^{a(-)}$, and $\Delta \mathrm{L}_{\sigma^{\prime} 0}=\mathrm{L}_{\sigma^{\prime} 0}^{(+)}-\mathrm{L}_{\sigma^{\prime} 0}^{(-)}$. Moreover, if there is no elastic constraint, we have $m^{\prime}=m^{a}=m^{b}=n-L$ $-l_{\mathcal{L}}$, and the jumps on the privileged velocities can be found from

$$
\Delta \dot{\phi}_{\sigma}=\sum_{\sigma^{\prime}=1}^{m^{\prime}}\left(\mathrm{M}^{-1}\right)_{\sigma \sigma^{\prime}}\left(-\Delta \mathrm{L}_{\sigma^{\prime} 0}+\mathrm{P}_{\sigma^{\prime}}^{(A)}\right), \quad \sigma=1, \cdots, m^{\prime} .
$$

With the prior quantities $\dot{\phi}_{\sigma}^{a(-)}$ being determined from the prior motion, the posterior quantities $\dot{\phi}_{\sigma}^{a(+)}$ are then computed from the previous equation, which, in turn, yield the posterior velocities of the system through Eq. (12).

After systematically discussing the impulsive motion associated with various impulsive constraints, the special case that the system is subject to applied impulses with only regular constraints being active is considered next. Since the kinematic conditions are the same for the prior, the virtual, and the posterior motion, the same set of privileged velocities can be chosen, denote by $\dot{\phi}_{1}, \cdots, \dot{\phi}_{m}$, with the same degree-of-freedom $m=n-L$. The corresponding coefficients for the velocity of each particle in three phases are thus the same, denoted by $\left\{\beta_{i \sigma}, \gamma_{i}\right\}_{i \in I, \sigma=1, \cdots, m}$. Similar to the special treatment on the live constraints, the privileged impulse-momentum equations become, cf. Eq. (32),

$$
\sum_{\sigma=1}^{m} \mathrm{M}_{\sigma^{\prime} \sigma} \Delta \dot{\phi}_{\sigma}=\mathrm{P}_{\sigma^{\prime}}^{(A)}, \quad \sigma^{\prime}=1, \cdots, m,
$$

since $\Delta \mathrm{L}_{\sigma^{\prime} 0}=0$ due to the nonexistence of live constraints. By computing the privileged applied impulses from the formula

$$
\mathrm{P}_{\sigma^{\prime}}^{(A)}=\sum_{i \in I} \mathbf{P}_{i}^{(A)} \cdot \bar{\beta}_{i \sigma^{\prime}}, \quad \sigma^{\prime}=1, \cdots, m,
$$

and the privileged mass matrix from

$$
\mathrm{M}_{\sigma \sigma^{\prime}}=\sum_{i \in I} m_{i} \bar{\beta}_{i \sigma} \cdot \bar{\beta}_{i \sigma^{\prime}}, \quad \sigma, \sigma^{\prime}=1, \cdots, m,
$$

the jumps on the privileged velocities can be determined from Eq. (34), and the posterior velocities of the system can be found subsequently from Eq. (12).

\section{Impulsive Motion for a System Containing Rigid Bodies}

A finite degree-of-freedom mechanical system $\mathcal{S}$ may be composed of many particles and rigid bodies. The physical quantities such as the virtual-privileged momenta and the virtual-privileged applied impulses can be defined for each subsystem. Due to the superposition property of the Eq. (23) with respect to the subsystems, a physical quantity for the system $\mathcal{S}$ can be obtained by taking the summation of the corresponding ones of all subsystems.

The motion of a rigid body $\mathcal{B}$ in $\mathcal{S}$ may be described by the translational motion of some reference point $Q$ in $\mathcal{B}$ and a rotation about $Q$, represented by the rotation dyadic $\Phi$. The rotation dyadic $\Phi$ changes the attitude of the rigid body from a reference (initial) configuration to the current configuration, and satisfies the property

$$
\Phi \cdot \Phi^{c}=1, \quad \operatorname{det} \Phi=1,
$$

where $\Phi^{c}$ denotes the conjugate of $\Phi$, and $l$ is the identity dyadic. It can be shown from the previous defining property that

$$
\dot{\Phi}=\boldsymbol{\omega} \times \Phi,
$$

in dyadic notation, [41], where $\boldsymbol{\omega}$ is the angular velocity of the rigid body. The position vector of any point $P \in \mathcal{B}$ can be then expressed as

$$
\mathbf{r}_{P}(t)=\mathbf{r}_{Q}(t)+\Phi(t) \cdot \mathbf{R}_{P Q},
$$

where $\mathbf{R}_{P Q}$ denotes the vector from $P$ to $Q$ at the reference configuration. Taking the time-derivative of the previous equation, we obtain

$$
\dot{\mathbf{r}}_{P}=\dot{\mathbf{r}}_{Q}+\boldsymbol{\omega} \times \Phi \cdot \mathbf{R}_{P Q}=\dot{\mathbf{r}}_{Q}+\boldsymbol{\omega} \times\left(\mathbf{r}_{P}-\mathbf{r}_{Q}\right) .
$$

Now let $\left(q_{1}, \cdots, q_{n}\right)$ be a set of generalized coordinates of $\mathcal{S}$. In general, the rotation dyadic $\Phi$ may be written in terms of the generalized coordinates as $\Phi=\Phi\left(q_{1}, \cdots, q_{n}, t\right)$, and its timederivative is

$$
\dot{\Phi}=\sum_{i=1}^{n} \frac{\partial \Phi}{\partial q_{i}} \dot{q}_{i}+\frac{\partial \Phi}{\partial t} .
$$

From the previous equation and Eq. (37), the expression of the angular velocity $\boldsymbol{\omega}$ in terms of the generalized velocities can be derived from the following identity:

$$
1 \times \boldsymbol{\omega}=\dot{\Phi} \cdot \Phi^{C}=\sum_{i=1}^{n}\left(\frac{\partial \Phi}{\partial q_{i}} \cdot \Phi^{C}\right) \dot{q}_{i}+\frac{\partial \Phi}{\partial t} \cdot \Phi^{C},
$$

as

$$
\boldsymbol{\omega}=\sum_{i=1}^{n} \hat{\boldsymbol{\omega}}_{i}(\mathbf{q}, t) \dot{q}_{i}+\hat{\boldsymbol{\omega}}_{0}(\mathbf{q}, t)
$$

In general, the right-hand side in the previous equation is not integrable, and the components of $\boldsymbol{\omega}$ with respect to certain frame can be treated as quasi-velocities of the system, cf. Eq. (7).

Consider the virtual motion of $\mathcal{S}$ during a constrained impulsive motion. With the appropriate selection of virtual-privileged velocities, $\dot{\phi}_{\sigma}^{\prime}$, the velocities of the reference point $Q$ and each particle $P$ in $\mathcal{B}$ can be expressed in the form of (11) with coefficients $\left(\gamma_{Q}^{\prime}, \beta_{Q 0}^{\prime}\right),\left(\beta_{P \sigma}^{\prime}, \gamma_{P}^{\prime}\right), \sigma=1, \cdots, m^{\prime}$, respectively, and the angular velocity of $\mathcal{B}$ is further written as

$$
\boldsymbol{\omega}=\sum_{\sigma=1}^{m^{\prime}} \boldsymbol{\varpi}_{\sigma}^{\prime} \dot{\phi}_{\sigma}^{\prime}+\varpi_{0}^{\prime} .
$$

Substituting these expressions into Eq. (38), it follows that

$$
\begin{aligned}
\sum_{\sigma=1}^{m^{\prime}}\left(\beta_{P \sigma}^{\prime}-\beta_{Q \sigma}^{\prime}\right) \dot{\phi}_{\sigma}^{\prime}+\left(\gamma_{P}^{\prime}-\gamma_{Q}^{\prime}\right) & \\
& =\sum_{\sigma=1}^{m^{\prime}} \varpi_{\sigma}^{\prime} \times\left(\mathbf{r}_{P}-\mathbf{r}_{Q}\right) \dot{\phi}_{\sigma}^{\prime}+\varpi_{0}^{\prime} \times\left(\mathbf{r}_{P}-\mathbf{r}_{Q}\right),
\end{aligned}
$$

which leads to

$$
\beta_{P \sigma}^{\prime}-\beta_{Q \sigma}^{\prime}=\varpi_{\sigma}^{\prime} \times\left(\mathbf{r}_{P}-\mathbf{r}_{Q}\right), \quad \gamma_{P}^{\prime}-\gamma_{Q}^{\prime}=\varpi_{0}^{\prime} \times\left(\mathbf{r}_{P}-\mathbf{r}_{Q}\right),
$$

for $\sigma=1, \cdots, m^{\prime}$, by the independency and the arbitrariness of the virtual-privileged velocities.

These relations, which based on the kinematic properties of a rigid body, are now applied to derive the prior and posterior virtual-privileged momenta, and virtual-privileged applied impulses for the rigid body $\mathcal{B}$ as follows. From Eqs. (19), (20), with the index set $I$ being over $P \in \mathcal{B}$, we have

$$
\begin{aligned}
\mathrm{L}_{\sigma}^{\mathcal{B}( \pm)} & \triangleq \int_{\mathcal{B}} \mathbf{v}_{P}^{( \pm)} \cdot \bar{\beta}_{P \sigma}^{\prime} d m(P) \\
& =\int_{\mathcal{B}} \mathbf{v}_{P}^{( \pm)} \cdot\left[\bar{\beta}_{Q \sigma}^{\prime}+\overline{\boldsymbol{\varpi}}_{\sigma}^{\prime} \times\left(\mathbf{r}_{P}-\mathbf{r}_{Q}\right)\right] d m(P) \\
& =\mathbf{L}^{\mathcal{B}( \pm)} \cdot \bar{\beta}_{Q \sigma}^{\prime}+\mathbf{H}_{Q}^{\mathcal{B}( \pm)} \cdot \overline{\boldsymbol{\varpi}}_{\sigma}^{\prime}, \quad \sigma=1, \cdots, m^{\prime},
\end{aligned}
$$




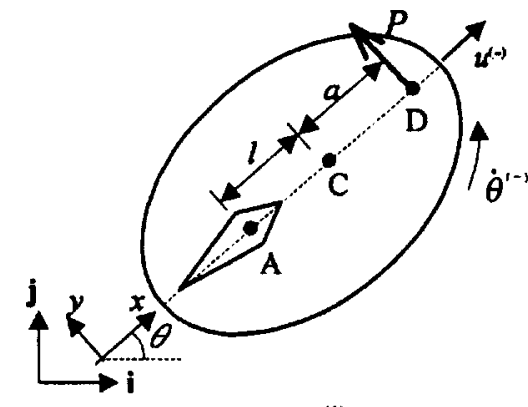

(i)

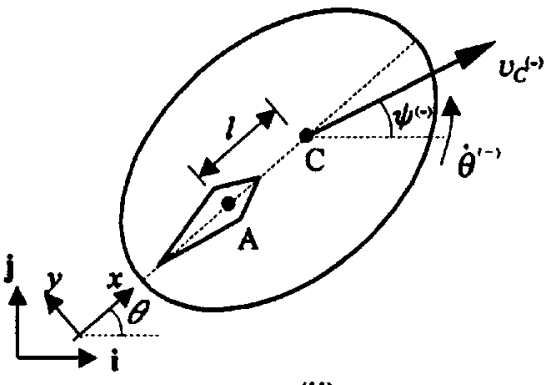

(ii)

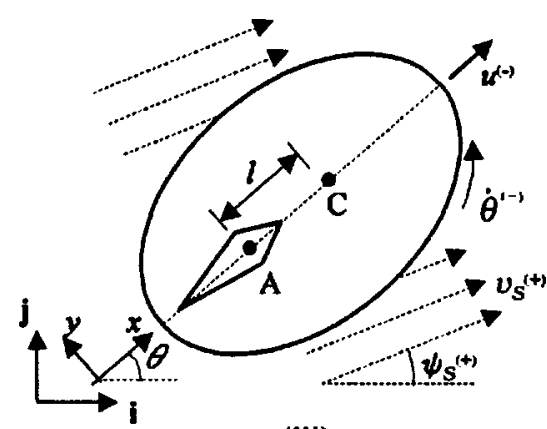

(iii)

Fig. 2 The impulsive motion of a sleigh with a knifeblade

$$
\mathbf{L}^{\mathcal{B}( \pm)}=\int_{\mathcal{B}} \mathbf{v}_{P}^{( \pm)} d m(P)
$$

are the prior and posterior linear momenta of $\mathcal{B}$, respectively, and

$$
\mathbf{H}_{Q}^{\mathcal{B}( \pm)}=\int_{\mathcal{B}}\left(\mathbf{r}_{P}-\mathbf{r}_{Q}\right) \times \mathbf{v}_{P}^{( \pm)} d m(P)
$$

are the prior and posterior angular momenta of $\mathcal{B}$ about the reference point $Q$, respectively. Let $C$ denote the center of mass of the rigid body $\mathcal{B}$, and $M, I_{Q}$ be the total mass and the moment of inertia dyadic of the rigid body about $Q$, respectively. The linear momenta and the angular momenta defined in (43), (44) can be further expressed as

$$
\begin{gathered}
\mathbf{L}^{\mathcal{B}( \pm)}=M \mathbf{v}_{C}^{( \pm)}, \\
\mathbf{H}_{Q}^{\mathcal{B}( \pm)}=I_{Q} \cdot \boldsymbol{\omega}^{( \pm)}+\left(\mathbf{r}_{C}-\mathbf{r}_{Q}\right) \times\left(M \mathbf{v}_{Q}^{( \pm)}\right),
\end{gathered}
$$

respectively.

On the other hand, the virtual-privileged applied impulses corresponding to $\mathcal{B}$ can be found to be, using Eq. (41),

$$
\begin{aligned}
\mathrm{P}_{\sigma}^{\mathcal{B}_{(A)}} & \triangleq \sum_{P \in \mathcal{B}} \mathbf{P}_{P}^{(A)} \cdot \bar{\beta}_{P \sigma}^{\prime}=\sum_{P \in \mathcal{B}} \mathbf{P}_{P}^{(A)} \cdot\left[\bar{\beta}_{Q \sigma}^{\prime}+\overline{\boldsymbol{\varpi}}_{\sigma}^{\prime} \times\left(\mathbf{r}_{P}-\mathbf{r}_{Q}\right)\right] \\
& =\mathbf{P}^{\mathcal{B}_{(A)}} \cdot \bar{\beta}_{Q \sigma}^{\prime}+\mathbf{J}_{Q}^{\mathcal{B}_{(A)}} \cdot \overline{\boldsymbol{\varpi}}_{\sigma}^{\prime}, \quad \sigma=1, \cdots, m^{\prime}
\end{aligned}
$$

where $\mathbf{P}^{\mathcal{B}_{(A)}}, \mathbf{J}_{Q}^{\mathcal{B}_{(A)}}$ are the resultant applied linear impulse, and the applied angular impulse acting on $\mathcal{B}$ about $Q$, defined as

$$
\begin{gathered}
\mathbf{P}^{\mathcal{B}_{(A)}}=\sum_{P \in \mathcal{B}} \mathbf{P}_{P}^{(A)}, \\
\mathbf{J}_{Q}^{\mathcal{B}_{(A)}}=\sum_{P \in \mathcal{B}}\left(\mathbf{r}_{P}-\mathbf{r}_{Q}\right) \times \mathbf{P}_{P}^{(A)},
\end{gathered}
$$

respectively.

After obtaining the formula for a single rigid body, the virtualprivileged momenta and applied impulses for the system consisting many bodies can be constructed. Let the system be composed of $N_{B}$ rigid bodies, which may be expressed as

$$
\mathcal{S}=\bigcup_{i=1}^{N_{B}} \mathcal{B}_{i}
$$

For each body $i, i=1, \ldots N_{B}$, we may choose a reference point $Q_{i}$, and denote the attitude dyadic by $\Phi_{i}$. With the selection of privileged velocities of $\mathcal{S}$ in the virtual motion, the coefficients $\left(\varpi_{\sigma}^{\prime}\right)^{\mathcal{B}_{i}}$ and $\beta_{Q_{i} \sigma}^{\prime}$ for each body can be determined. The virtualprivileged momenta and the applied impulses of the system are then found from the following summation:

$$
\mathrm{L}_{\sigma}^{( \pm)}=\sum_{i=1}^{N_{B}} \mathrm{~L}_{\sigma}^{\mathcal{B}_{i}( \pm)}, \quad \mathrm{P}_{\sigma}^{(A)}=\sum_{i=1}^{N_{B}} \mathrm{P}_{\sigma}^{\mathcal{B}_{i(A)}},
$$

respectively. With these formulas, the privileged impulsemomentum equations developed in Section 4 can be invoked to solve the constrained impulsive problems associated with interconnected rigid bodies. An example is given in the next section to illustrate the process.

\section{The Impulsive Motion of a Sleigh With a Knifeblade}

The proposed methodology discussed in the previous sections is now applied to study the impulsive motion of a sleigh with a knifeblade attached. Three possibilities of impulsive motions due to applied impulse, inert constraint, and live constraint, respectively, are discussed. Two alternative methods, namely, Newton's method and Lagrange's method, are used to solve the same problem for the case of inert constraint to describe the essential differences.

Consider a sleigh $\mathcal{B}$ with a knifeblade along its principle axis, as depicted in Fig. 2. Let its mass and moment of inertia about its center of mass $C$ be denoted by $M$ and $I_{C}$, respectively. When the sleigh moves on a horizontal ice surface $S$, it is assumed that the knifeblade makes contact with the surface at point $A$, and it is possible for the sleigh to move freely in the direction along the blade, and rotate freely about $A$. However, the motion of the sleigh in the direction perpendicular to the blade is prohibited. Let $\{\mathbf{i}, \mathbf{j}\}$ be a fixed coordinate system, and $\left\{\mathbf{e}_{x}, \mathbf{e}_{y}\right\}$ be a coordinate system moving with $\mathcal{B}$, where $\mathbf{e}_{x}$ is parallel to the direction of the blade, which makes an angle $\theta$ with respect to the axis i. Due to the physical condition of constraints, the velocity of $A$ satisfies

$$
\mathbf{v}_{A} \cdot \mathbf{e}_{y}=0,
$$

and may be expressed as

$$
\mathbf{v}_{A}=u \mathbf{e}_{x},
$$

where $u$ is termed the longitudinal velocity of $\mathcal{B}$. The sleigh may rotate about the vertical axis $\mathbf{e}_{z}\left(=\mathbf{e}_{x} \times \mathbf{e}_{y}\right)$ with angular velocity $\dot{\theta} \mathbf{e}_{z}$. The velocity of $C$ may be then expressed as

$$
\mathbf{v}_{C}=u \mathbf{e}_{x}+l \dot{\theta} \mathbf{e}_{y},
$$

where $l$ denotes the distance between $C$ and $A$. For this problem, we may choose the coordinates of $C$, i.e., $\left(x_{C}, y_{C}\right)$, and the angle $\theta$ as the generalized coordinates. The constraints (51) then becomes

$$
-\sin \theta \dot{x}_{C}+\cos \theta \dot{y}_{C}-l \dot{\theta}=0,
$$

which is nonholonomic. The longitudinal velocity $u$ can be expressed as

$$
u=\cos \theta \dot{x}_{C}+\sin \theta \dot{y}_{C},
$$


in which the right-hand side is not integrable, and thus should be treated as a quasi-velocity.

We are now ready to consider three cases of impulsive motions for this system, which occur at the instant $\bar{t}$ and at the configuration of $\theta=\theta_{0}$.

Case (i). As shown in Fig. 2(i), the sleigh is suddenly exerted by an impulse $P$ acting at the point $D$ at the instant $\bar{t}$. The longitudinal velocity of $\mathcal{B}$ and the angular velocity of $\mathcal{B}$ about the vertical axis at the instant $\bar{t}-$ are $u^{(-)}$and $\dot{\theta}^{(-)}$, respectively. It is desired to determine these two quantities at the instant $\bar{t}+$.

For this case, the impulsive motion is due to the imposition of the applied impulse $P$, while the rigid body $\mathcal{B}$ is subject to the regular nonholonomic constraint, Eq. (54). One may choose the longitudinal velocity $u$ and the angular velocity $\dot{\theta}$ as the privileged velocities to represent the possible motion of $\mathcal{B}$. In fact, the velocity of $A$ and $C$ can be written in terms of the privileged velocities as in Eqs. (52) and (53), respectively. Since the prior and the posterior motions satisfy the same nonholonomic constraint, the prior and posterior velocities of $A$ and $C, \mathbf{v}_{A}^{(-)}, \mathbf{v}_{C}^{(-)}, \mathbf{v}_{A}^{(+)}$and $\mathbf{v}_{C}^{(+)}$, can be also expressed in terms of the prior and posteriorprivileged velocities, $u^{(-)}, \dot{\theta}^{(-)}, u^{(+)}$, and $\dot{\theta}^{(+)}$, with the same form, respectively.

Let $C$ be selected as the reference point for $\mathcal{B}$. The coefficients of the velocity of $C$ and the angular velocity of $\mathcal{B}$ corresponding to the privileged velocities can be obtained as

$$
\begin{array}{cc}
\bar{\beta}_{C 1}=\mathbf{e}_{x}, & \bar{\beta}_{C 2}=l \mathbf{e}_{y}, \\
\bar{\varpi}_{1}=0, & \bar{\varpi}_{2}=\mathbf{e}_{z},
\end{array}
$$

respectively. The linear momenta, and the angular momenta about $C$ given in (43), (44), are then

$$
\begin{gathered}
\mathbf{L}^{\mathcal{B}( \pm)}=M u^{( \pm)} \mathbf{e}_{x}+M l \dot{\theta}^{( \pm)} \mathbf{e}_{y}, \\
\mathbf{H}_{C}^{\mathcal{B}( \pm)}=I_{C} \dot{\theta}^{( \pm)} \mathbf{e}_{z},
\end{gathered}
$$

respectively. On the other hand, the applied linear impulse and the applied angular impulse are, respectively,

$$
\mathbf{P}^{\mathcal{B}_{(A)}}=P \mathbf{e}_{y}, \quad \mathbf{J}_{C}^{\mathcal{B}_{(A)}}=P a \mathbf{e}_{z} .
$$

Substituting these terms into the formula of the privileged momenta and privileged applied impulses, the privileged impulsemomentum Eq. (18) can be applied to derive, in matrix form,

$$
[\mathrm{M}]\left(\left[\begin{array}{c}
u^{(+)} \\
\dot{\theta}^{(+)}
\end{array}\right]-\left[\begin{array}{c}
u^{(-)} \\
\dot{\theta}^{(-)}
\end{array}\right]\right)=\left[\begin{array}{c}
0 \\
P(a+l)
\end{array}\right],
$$

where the privileged mass matrix $[\mathrm{M}]$ is given by

$$
[\mathrm{M}]=\left[\begin{array}{cc}
M & 0 \\
0 & \left(M l^{2}+I_{C}\right)
\end{array}\right] .
$$

Hence, the posterior longitudinal velocity and angular velocity of the sleigh $\mathcal{B}$ can be determined as

$$
\left[\begin{array}{c}
u^{(+)} \\
\dot{\theta}^{(+)}
\end{array}\right]=[\mathrm{M}]^{-1}\left[\begin{array}{c}
0 \\
P(a+l)
\end{array}\right]+\left[\begin{array}{c}
u^{(-)} \\
\dot{\theta}^{(-)}
\end{array}\right]=\left[\begin{array}{c}
u^{(-)} \\
\frac{a+l}{M l^{2}+I_{C}} P+\dot{\theta}^{(-)}
\end{array}\right] .
$$

Case (ii). Consider the landing of the sleigh on the ice surface at the instant $\bar{t}$, with prior velocity of the center of mass being $v_{C}^{(-)}$ in the direction of angle $\psi^{(-)}$with respect to the axis $\mathbf{i}$, and the prior angular velocity about $\mathbf{e}_{z}$ being $\dot{\theta}^{(-)}$, cf. Fig. 2(ii). The velocity of the landing point $A$ may have lateral component before $\bar{t}$, but it immediately satisfies the constraint (51) after contact. This impulsive constraint is thus characterized as an inert constraint. The virtual motion and the posterior motion have the same characteristics as the motion in Case (i). Similar to the discussion there, the set of velocities $(u, \dot{\theta})$ may be chosen as the virtualprivileged velocities, $\dot{\phi}_{1}^{\prime}=u, \dot{\phi}_{2}^{\prime}=\dot{\theta}$. However, they are not the privileged velocities for the prior motion. Again, the point $C$ is chosen as the reference point for $\mathcal{B}$, and the coefficients for the velocity of $C$ and the angular velocity of $\mathcal{B}$ corresponding to the virtual-privileged velocities are the same as in (55), with $\bar{\beta}, \bar{\varpi}$ being replaced by $\bar{\beta}^{\prime}, \bar{\varpi}^{\prime}$ respectively. The required quantities can be found to be

$$
\begin{aligned}
& \mathbf{L}^{\mathcal{B}_{(-)}}=M v_{C}^{(-)} \cos \left(\psi^{(-)}-\theta_{0}\right) \mathbf{e}_{x}+M v_{C}^{(-)} \sin \left(\psi^{(-)}-\theta_{0}\right) \mathbf{e}_{y}, \\
& \mathbf{L}^{\mathcal{B}_{(+)}}=M u^{(+)} \mathbf{e}_{x}+M l \dot{\theta}^{(+)} \mathbf{e}_{y}, \\
& \mathbf{H}_{C}^{\mathcal{B}_{( \pm)}}=I_{C} \dot{\theta}^{( \pm)} \mathbf{e}_{z} \text {. }
\end{aligned}
$$

The prior and the posterior virtual-privileged momenta corresponding respectively to $\phi_{1}^{\prime}, \phi_{2}^{\prime}$ are then

$$
\begin{gathered}
\mathrm{L}_{1}^{(-)}=M v_{C}^{(-)} \cos \left(\psi^{(-)}-\theta_{0}\right), \\
\mathrm{L}_{1}^{(+)}=M u^{(+)}, \\
\mathrm{L}_{2}^{(-)}=M l v_{C}^{(-)} \sin \left(\psi^{(-)}-\theta_{0}\right)+I_{C} \dot{\theta}^{(-)}, \\
\mathrm{L}_{2}^{(+)}=\left(M l^{2}+I_{C}\right) \dot{\theta}^{(+)} .
\end{gathered}
$$

Since there is no applied impulse, the conservation of virtualprivileged momenta can be then invoked to yield the posterior velocities as

$$
\begin{gathered}
u^{(+)}=v_{C}^{(-)} \cos \left(\psi^{(-)}-\theta_{0}\right), \\
\dot{\theta}^{(+)}=\frac{1}{M l^{2}+I_{C}}\left(M l v_{C}^{(-)} \sin \left(\psi^{(-)}-\theta_{0}\right)+I_{C} \dot{\theta}^{(-)}\right) .
\end{gathered}
$$

Case (iii). If the ice surface exhibits a sudden motion at $\bar{t}$ caused by external agents, such as an earthquake, the sleigh moving on it then experiences an impulsive constraint. Let the velocity of the surface be denoted by $\mathbf{v}_{S}$. Instead of (51), the constraint becomes now

$$
\left(\mathbf{v}_{A}-\mathbf{v}_{S}\right) \cdot \mathbf{e}_{y}=0
$$

or

$$
\mathbf{v}_{A} \cdot \mathbf{e}_{y}-\mathbf{v}_{S} \cdot \mathbf{e}_{y}=0,
$$

in which the inhomogeneous term makes it become a live impulsive constraint. In particular, let the surface suddenly move with velocity $v_{S}^{(+)}$in the direction of angle $\psi_{S}^{(+)}$with respect to the axis i. The velocity of $A$ and $C$ during the impulsive motion can be then expressed as

$$
\begin{gathered}
\mathbf{v}_{A}=u \mathbf{e}_{x}+v_{S}\left(\cos \psi_{S} \mathbf{i}+\sin \psi_{S} \mathbf{j}\right), \\
\mathbf{v}_{C}=u \mathbf{e}_{x}+l \dot{\theta} \mathbf{e}_{y}+v_{S}\left(\cos \psi_{S} \mathbf{i}+\sin \psi_{S} \mathbf{j}\right),
\end{gathered}
$$

respectively, in which $u$ is now the longitudinal velocity of the sleigh relative to the surface, and $v_{S}, \psi_{S}$ are the magnitude and the direction of $\mathbf{v}_{S}$, respectively. From the constraint, we have

$$
\begin{array}{ll}
v_{S}(\bar{t}-)=0, & v_{S}(\bar{t}+)=v_{S}^{(+)}, \\
\psi_{S}(\bar{t}-)=0, & \psi_{S}(\bar{t}+)=\psi_{S}^{(+)} .
\end{array}
$$

For this case, the prior motion, the virtual motion, and the posterior motion have the same degree-of-freedom, and the same set of privileged velocities $\dot{\phi}_{1}^{\prime}=u, \dot{\phi}_{2}^{\prime}=\dot{\theta}$ may be chosen. Again, Eq. (55), after replacing $\bar{\beta}$, $\bar{\varpi}$ by $\bar{\beta}^{\prime}, \bar{\varpi}^{\prime}$, is applicable, and the quantities of momenta for the body are found as

$$
\begin{aligned}
& \mathbf{L}^{\mathcal{B}_{(-)}}=M\left(u^{(-)} \mathbf{e}_{x}+l \dot{\theta}^{(-)} \mathbf{e}_{y}\right), \\
& \mathbf{L}^{\mathcal{B}_{(+)}}=M\left[u^{(+)} \mathbf{e}_{x}+l \dot{\theta}^{(+)} \mathbf{e}_{y}+v_{S}^{(+)}\left(\cos \psi_{S}^{(+)} \mathbf{i}+\sin \psi_{S}^{(+)} \mathbf{j}\right)\right],
\end{aligned}
$$




$$
\mathbf{H}_{C}^{\mathcal{B}_{( \pm)}}=I_{C} \dot{\theta}^{( \pm)} \mathbf{e}_{z}
$$

These formulas in turn give rise to the prior and the posterior virtual-privileged momenta corresponding to $\dot{\phi}_{1}^{\prime}, \dot{\phi}_{2}^{\prime}$ as

$$
\begin{gathered}
\mathrm{L}_{1}^{(-)}=M u^{(-)}, \\
\mathrm{L}_{1}^{(+)}=M u^{(+)}+M v_{s}^{(+)} \cos \left(\psi_{s}^{(+)}-\theta_{0}\right), \\
\mathrm{L}_{2}^{(-)}=\left(M l^{2}+I_{C}\right) \dot{\theta}^{(-)}, \\
\mathrm{L}_{2}^{(+)}=\left(M l^{2}+I_{C}\right) \dot{\theta}^{(+)}+M l v_{s}^{(+)} \sin \left(\psi_{s}^{(+)}-\theta_{0}\right),
\end{gathered}
$$

respectively. The conservation of the virtual-privileged momenta is then invoked to find the posterior longitudinal velocity relative to the surface and the posterior angular velocity as

$$
\begin{gathered}
u^{(+)}=u^{(-)}-v_{s}^{(+)} \cos \left(\psi_{s}^{(+)}-\theta_{0}\right), \\
\dot{\theta}^{(+)}=\dot{\theta}^{(-)}-\frac{M l}{M l^{2}+I_{C}} v_{s}^{(+)} \sin \left(\psi_{s}^{(+)}-\theta_{0}\right) .
\end{gathered}
$$

To show the distinct features of the proposed scheme, Newton's method and Lagrange's method for impulsive motion are applied next to solve the same problem as in Case (ii).

As discussed in [2], the integration of Newton-Euler's equations gives rise to the law of impulse for impulsive motion. For the problem of Case (ii), the only impulsive force acting on $\mathcal{B}$ is the one acting on $A$ exerted by the ice surface, which has no component in the direction perpendicular to the blade by the nature of the constraint. Accordingly, we have the conservation of linear momentum in that direction,

$$
\mathbf{L}^{(+)} \cdot \mathbf{e}_{x}=\mathbf{L}^{(-)} \cdot \mathbf{e}_{x},
$$

or

$$
M u^{(+)}=M v_{C}^{(-)} \cos \left(\psi^{(-)}-\theta_{0}\right) .
$$

On the other hand, there is no impulsive torque about $A$ either, and the conservation of angular momentum is then

$$
I_{C} \dot{\boldsymbol{\theta}}^{(+)} \mathbf{e}_{z}+\left(\mathbf{r}_{C}-\mathbf{r}_{A}\right) \times\left(M \mathbf{v}_{C}^{(+)}\right)=I_{C} \dot{\theta}^{(-)} \mathbf{e}_{z}+\left(\mathbf{r}_{C}-\mathbf{r}_{A}\right) \times\left(M \mathbf{v}_{C}^{(-)}\right) .
$$

From the relation $\mathbf{v}_{C}^{(+)}=u^{(+)} \mathbf{e}_{x}+l \dot{\theta}^{(+)} \mathbf{e}_{y}$, the posterior longitudinal velocity and posterior angular velocity can be obtained exactly the same as in Eq. (57).

Although Newton's method seems to be more intuitive for this simple case, it is not straightforward to apply the method to more sophisticated problems. For example, to handle the impulsive problems for constrained multibody systems, it is rather complicated to solve the problems by inspection.

On the other hand, in Lagrange's method, cf. [3,5,18], the equations of impulsive motion are written in terms of the kinetic energy $T$ of the system. The problem studied in Case (i) has been solved by Lagrange's method in [18]. Here, the same method is applied to attack the impulsive problem for Case (ii), and is summarized as follows. Consider the system with kinetic energy $T$, and subject to $l_{\mathcal{I}}$ inert impulsive constraints at $\bar{t}$. From the impulsive constraints, $n-l_{\mathcal{I}}$ generalized velocities, $\dot{q}_{1}, \cdots, \dot{q}_{n-l_{\mathcal{I}}}$, can be selected to represent the other ones such that

$$
\dot{q}_{j}=\sum_{\sigma=1}^{n-l_{I}} D_{j \sigma} \dot{q}_{\sigma}+d_{j}, \quad j=n-l_{\mathcal{I}}+1, \ldots, n .
$$

Then the prior motion and the posterior motion satisfy the Lagrange's equations for impulsive motion, i.e., for $\sigma=1, \cdots, n$ $-l_{\mathcal{I}}$

$\left.\left(\frac{\partial T}{\partial \dot{q}_{\sigma}}+\sum_{j=n-l_{x}+1}^{n} \frac{\partial T}{\partial \dot{q}_{j}} D_{j \sigma}\right)\right|_{\bar{t}+}=\left.\left(\frac{\partial T}{\partial \dot{q}_{\sigma}}+\sum_{j=n-l_{x}+1}^{n} \frac{\partial T}{\partial \dot{q}_{j}} D_{j \sigma}\right)\right|_{\bar{t}-}$.
For the previous example, with the generalized coordinates $\left(x_{c}, y_{c}, \theta\right)$, the kinetic energy of the sleigh is simply

$$
T=\frac{1}{2} M\left(\dot{x}_{C}^{2}+\dot{y}_{C}^{2}\right)+\frac{1}{2} I_{C} \dot{\theta}^{2} .
$$

The inert constraint (54) may be expressed as

$$
\dot{\theta}=-\frac{\sin \theta}{l} \dot{x}_{C}+\frac{\cos \theta}{l} \dot{y}_{C} .
$$

Corresponding to the generalized coordinates $x_{c}, y_{c}$, the equations of the impulsive motion can be derived from (61) as

$$
\begin{aligned}
& M \dot{x}_{C}^{(+)}-\frac{I_{C}}{l} \dot{\theta}^{(+)} \sin \theta_{0}=M \dot{x}_{C}^{(-)}-\frac{I_{C}}{l} \dot{\theta}^{(-)} \sin \theta_{0}, \\
& M \dot{y}_{C}^{(+)}+\frac{I_{C}}{l} \dot{\theta}^{(+)} \cos \theta_{0}=M \dot{y}_{C}^{(-)}+\frac{I_{C}}{l} \dot{\theta}^{(-)} \cos \theta_{0} .
\end{aligned}
$$

The prior velocity of $C$ is $\left(v_{C}^{(-)} \cos \psi^{(-)}, v_{C}^{(-)} \sin \psi^{(-)}\right)$, and the posterior velocity can be expressed in terms of the posterior longitudinal velocity and angular velocity as

$$
\begin{aligned}
& \dot{x}_{C}^{(+)}=\cos \theta_{0} u^{(+)}-l \sin \theta_{0} \dot{\theta}^{(+)}, \\
& \dot{y}_{C}^{(+)}=\sin \theta_{0} u^{(+)}+l \cos \theta_{0} \dot{\theta}^{(+)} .
\end{aligned}
$$

Substituting the above formulas into (63), the posterior velocities $u^{(+)}, \dot{\theta}^{(+)}$can be then determined by exactly the same equations as (57).

It is noted that in Lagrange's method, the quasi-velocity $u$ is not introduced directly, which reflects the fact that the treatment of quasi-velocity is a little awkward in Lagrange's formulation. Moreover, the computation of kinetic energy can only accommodate the regular constraints. If the impulsive constraints occur, additional terms have to be included in the equations. Since the proposed methodology somewhat has similar characters as the Kane's approach for the finite-force problem, the comparison of different methods in [28] can be partly transported here. In particular, it has been shown in $[27,42]$ that Kane's approach is very effective in generating equations of motion for multibody systems through symbolic computations. The same advantage of applying the proposed method to deal with impulsive motion of multibody systems subject to impulsive constraints is conceivable accordingly. Using the method to tackle the impulsive constraints, it is not necessary to include the impulsive constraint forces in the equations, which may contain some parameters, e.g., Lagrange's multipliers, to be determined in the process.

\section{Conclusions}

Although there are many approaches dealing with the constrained impulsive problems for mechanical systems, the principle of velocity variation for impulsive motions lays down the foundation of the methodology discussed in this paper. The classical principles for finite-force problems may not be suitable to be applied directly to the impulsive problems, due to the irregularity of the occurrences of the impulsive constraints. From the fundamental principle, it was shown that, instead of the classical notion of conservation of momenta for systems without applied impulses, the more appropriate law should be the conservation of virtualprivileged momenta, respecting the behavior of the system in the virtual motion. With the inclusion of quasi-velocities, such as the angular velocity of a rigid body, or the longitudinal velocity of a vehicle, the selection of independent privileged velocities seems to be more natural. The methodology discussed in this paper provides a systematic tool in dealing with various types of impulsive constraints, and can be applied to solve a variety of constrained impulsive problems for mechanical systems. 


\section{References}

[1] Appell, P., 1923, Traité de Mécanique Rationnelle, Vol. 2, 4th Ed., GauthierVillars, Paris.

[2] Whittaker, E. T., 1937, A Treatise on the Analytical Dynamics of Particles and Rigid Bodies, Cambridge, UK.

[3] Pars, L. A., 1965, A Treatise on Analytical Dynamics, Heinemann, London.

[4] Goldstein, H., 1980, Classical Mechanics, Addison-Wesley, Redding, MA.

[5] Rosenberg, R. M., 1977, Analytical Dynamics of Discrete Systems, Plenum Press, New York.

[6] Meirovitch, L., 1970, Methods of Analytical Dynamics, McGraw-Hill, New York.

[7] Kane, T. R., 1962, “Impulsive Motions,” ASME J. Appl. Mech., 29, pp. $715-$ 718.

[8] Papastavridis, J. G., 1989, “Impulsive Motion of Ideally Constrained Mechanical Systems via Analytical Dynamics," Int. J. Eng. Sci., 27(12), pp. $1445-1461$.

[9] Stronge, W. J., 1990, "Rigid Body Collision With Friction," Proc. R. Soc. London, Ser. A, 431, pp. 169-181.

[10] Souchet, R., 1993, "Analytical Dynamics of Rigid Body Impulsive Motions," Int. J. Eng. Sci., 31(1), pp. 85-92.

[11] Bahar, L. Y., 1994, "On The Use of Quasi-Velocities in Impulsive Motion," Int. J. Eng. Sci., 32(11), pp. 1669-1686.

[12] Ibort, A., de Leon, M., Lacomba, E. A., de Diego, D. Martin, and Pitanga, P., 1997, "Mechanical Systems Subjected to Impulsive Constraints," J. Phys. A, 30, pp. 5835-5854.

[13] Pfeiffer, F., and Glocker, C., 1996, Multibody Dynamics With Unilateral Contacts, John Wiley and Sons, New York.

[14] Brogliato, B., 1996, Nonsmooth Impact Mechanics: Models, Dynamics, and Control, Springer-Verlag, London.

[15] Lacomba, E. A., and Tulczyjew, W. M., 1990, "Geometric Formulation of Mechanical Systems With One-sided Constraints," J. Phys. A, 23, pp. 28012813.

[16] Genot, F., and Brogliato, B., 1999, "New Results on Painleve Paradoxes," Eur. J. Mech. A/Solids, 18, pp. 653-677.

[17] van der Schaft, A. J., and Schumacher, J. M., 1999, "Complementarity Modeling of Hybrid Systems,” IEEE Trans. Autom. Control, 43(4), pp. 483-490.

[18] Neimark, Ju. I., and Fufaev, N. A., 1972, Dynamics of Nonholonomic Systems (Vol. 33 of Translations of Mathematical Monographs) American Mathematical Society, Providence, RI.

[19] Greenwood, D. T., 1988, Principles of Dynamics, Prentice-Hall, Englewood Cliffs, NJ.

[20] Ibort, A., de Leon, M., Lacomba, E. A., Marrero, J. C., Martin de Diego, D. and Pitanga, P., 1998, "Geometric Formulation of Mechanical Systems Subjected to Time-Dependent One-Sided constraints," J. Phys. A, 31, pp. 26552674.
[21] Cortés, J., de León, M., de Diego, D. Martín, and Martínez, S., 2001, "Mechanical Systems Subjected to Generalized Non-holonomic Constraints," Proc. R. Soc. London, Ser. A, 457, pp. 651-670.

[22] Jourdain, P. E. B., 1909, "Note on an Analogue of Gauss' Principle of Least Constraint," Quart. J. Pure Appl. Math., 40, pp. 153-157.

[23] Roberson, R. E., and Schwertassek, R., 1988, Dynamics of Multibody Systems, Springer-Verlag, New York.

[24] Moon, F. C., 1998, Applied Dynamics, John Wiley and Sons, New York.

[25] Wang, L. S., and Pao, Y. H., 2003, "Jourdain's Variational Equation and Appell's Equation of Motion for Nonholonomic Dynamical Systems," Am. J. Phys., 73(1), pp. 72-82.

[26] Pao, Y. H., and Wang, L. S., 2002, "A Unified Variational Principle for Dynamics of Particles and of Continua," submitted for journal publication.

[27] Lesser, M., 1995, The Analysis of Complex Nonlinear Mechanical Systems: A Computer Algebra Assisted Approach, World Scientific, Singapore.

[28] Kane, T. R., and Levinson, D. A., 1980, "Formulation of Equations of Motion for Complex Spacecraft," AIAA J. Guidance Control, 3(2), pp. 99-112.

[29] Scott, D., 1988, "Can a Projection Method of Obtaining Equations of Motion Compete With Lagrange's Equations?” Am. J. Phys., 56, pp. 451-456.

[30] Blajer, W., 1992, "A Projection Method Approach to Constrained Dynamic Analysis," ASME J. Appl. Mech., 59, pp. 643-649.

[31] Ibort, A., de Leon, M., Lacomba, E. A., Marrero, J. C., de Diego, D. Martin, and Pitanga, P., 2001, "Geometric Formulation of Carnot's Theorem," J. Phys. A, 34, pp. 1691-1712

[32] Arnold, V. I., 1989, Mathematical Methods of Classical Mechanics, 2nd Ed., Springer-Verlag, New York.

[33] Abraham, R., and Marsden, J. E., 1978, Foundations of Mechanics, 2nd Ed. Benjamin/Cummings, Reading, MA.

[34] Vershik, A. M., and Faddeev, L. D., 1981, "Lagrangian Mechanics in Invariant Form," Sel. Math. Sov., 1(4), pp. 339-350.

[35] Tulczyjew, W. M., 1989, Geometric Formulation of Physical Theories: Statics and Dynamics of Mechanical Systems, Bibliopolis, Naples.

[36] Gibbs, J. W., 1879, "On the Fundamental Formulas of Dynamics," Am. J. Math., 2, pp. 49-64.

[37] Mach, E., 1960, The Science of Mechanics, Open Court, La Salle, IL.

[38] Arnold, V. I., ed., 1988, Dynamical Systems III (Vol. 3 of Encyclopedia of Mathematical Sciences), Springer-Verlag, New York.

[39] Chetaev, N. G., 1932, "On the Principle of Gauss," Kazan Univ. Bull. Phys. Math. Soc., 6, pp. 68-71.

[40] Smith, C. E., and Liu, P. P., 1992, "Coefficients of Restitution," ASME J. Appl. Mech., 59, pp. 963-969.

[41] Weatherburn, C. E., 1921, Elementary Vector Analysis, Open Court, La Salle, IL.

[42] Junkins, John L., ed., 1990, Mechanics and Control of Large Flexible Structures, American Institute of Aeronautics and Astronautics, Washington, DC. 